anayake. One is that there was some problem with the analytical procedure used in the original report. Aluminium measurements are difficult to make and errors are extremely common ${ }^{3}$. Second, Tennakone and Wickramanayake might have used a different form of fluoride such as hydrogen fluoride. Third, there might be some differences in the aluminium pans. However, we chose many different types and styles of cookware and detected no significant differences in the degree of aluminium leaching.

In view of the concern that the report by Tennakone and Wickramanayake has raised among those interested in water fluoridation, we would like to emphasize that we cannot reproduce the results in that report.

J. SAVORY

J.R. NICHOLSON

M.R. WIL.LS

University of Virginia Medical Center,

Departments of Pathology, Biochemistry and Internal Medicine,

Charlottesville,

Virginia 22908, USA

1. Tennakonc, K. \& Wickramanayake, S. Nature 325. 202 (1987)

Brown. S., Bertholf, R.L., Wills, M.R. \& Savory. J. Clin (hem. 30, $1216(1984)$

Cornclis, R. \& Schutyser, P. Contr. Nephrol. 38. 1 (1984).

\section{Cosmic strings and celestial objects}

SIR-In a recent paper ${ }^{1}$, Tassie has suggested that celestial objects have evolved through a hierarchical breaking of rotating pieces of cosmic string and that this would account for the angular momentum $(J)$ for a wide variety of astronomical objects being proportional to the square of their masses, $(M)$, the constant of proportionality moreover being comparable with that of some string theories of particle physics. So one has a relation $J=K M^{2}, K$ being a universal constant whose value from an analysis of astronomical objects of different classes is $\sim 10^{-15} \mathrm{~g}^{-1} \mathrm{~cm}^{2} \mathrm{~s}^{-1}$ and similarly for strings $J_{\mathrm{S}} \approx K_{\mathrm{S}} M^{2}$ with $K_{\mathrm{S}}$ being related to the string tension $\mu$ through $K_{\mathrm{S}}=c(2 \pi \mu)^{-1}$. For agreement with the astronomical value, that is $K=$ $K_{\mathrm{S}}$, this would imply a string tension of $\mu \approx 6 \times 10^{24} \mathrm{~g} \mathrm{~cm}^{-1}$. It must first be pointed out that it is not necessary to invoke exotic configurations such as strings to understand the apparent $J=K M^{2}$ relation for celestial objects. In fact it can be shown ${ }^{2}$ that just such a relation with a dimensional constant $K$ close to the observed value, would arise naturally from considerations of the basic physics involved in the structure of various astronomical objects such as galaxies and stars. The relation in fact is an upper envelope relating maximum $J$ to $M^{2}$. To within a factor of order unity, systems which are gravitationally bound have typical velocities $(V / c) \approx\left(R_{\mathrm{G}} / R\right)^{1 / 2}$, $R_{\mathrm{G}}$ being the gravitational radius $\left(2 G M / c^{2}\right)$. If $V^{2} / R \approx G M / R^{2}$ and $J=$ $M V R$ we can write $J \approx(G / V) M^{2}$ which for $V \approx$ constant would imply an apparent $J \sim M^{2}$ relation. It is to be noted that the range of $V$ or equivalently $\left(R_{\mathrm{G}} / R\right)^{1 / 2}$ is rather narrow for astronomical objects, (as compared to the range of $M$ ), with $10^{-3} c$ being most typical. (Velocity $V$ well below $10^{-4} c$ would be very difficult to observe and relativistic objects are rare.) Thus for $V \approx 10^{-3} c, K \approx 10^{-15}$, which is the empirical result seen.

Apart from the fact that there is no need to invoke strings to account for the $J=$ $K M^{2}$ relation, the value for the string tension $\mu$, which the observed value of $K$ would imply is unacceptably high. The reason being that the dominant energy loss mechanism for strings is by gravitational radiation with the power loss given by $P=\beta G \mu^{2} c, \beta \approx 10$. This rate of energy loss is independent of the size of the string (it depends only on the tension $\mu$ ). For a string of radius $R$ this implies a lifetime $t \approx R C / \beta G \mu$. For galaxy-size loops $R \approx 1$ $\mathrm{kpc}$, and with the above value of $\mu$, this would give a lifetime of less than one per cent of a Hubble time, that is, they would not last long enough to form galaxies. A more stringent constraint ${ }^{3}$ (C.S., in preparation) obtained by noting that the presence of an extra component (of gravitational radiation) or even less than $5 \%$ of the total radiation background at the epoch of nucleosynthesis can affect element abundances, give $\mu<10^{20} \mathrm{~g} \mathrm{~cm}^{-1}$. This makes it improbable that the value of $\mu$ determines the constant of proportionality in the $J-M^{2}$ relation; that is, the value of $\mu$ implied from this relation being incompatible with string models of galaxy formation.

Indian Institute of Astrophysics, Bangalore 560034, India

1. Tassie, L. J. Nature 323, 40-43 (1986).

2. Sivaram, C. in Relativistic Astrophysics and Cosmology (ed. de Sabbatta, V.) 228-246 (World Scientific, Singapore 1984)

TASSIE REPLIES-Defining a velocity $V$ by $V=G M^{2} / J$, then, as Sivaram points out, $V$ constant would imply an apparent $J=K M^{2}$ relation. But this raises the question of why $V$ should be so constant for astronomical objects. The string picture ${ }^{1}$ explains the constancy of both $J / M^{2}$ and $V$. Sivaram's $V$ is a measure of the velocity in gravitationally bound systems, such as binary stars, but is not simply related to the rotational velocity of a planet such as Jupiter with a rotational velocity at the equator of $4 \times 10^{-5} \mathrm{c} . J / \mathrm{M}^{2}$ for Jupiter, however, is comparable with that of flat galaxies ${ }^{1}$.

Sivaram's second point, concerning the effect of gravitational radiation, is important as it yields a bound on the time for string fragmentation. Witten ${ }^{2}$ has pointed out that Type 1 superstrings fragment too fast to contribute cosmologically significant gravitation radiation. As the theory of the superstring includes gravitational radiation, which is the emission of massless closed loops by the string, and because the thermodynamic treatment of string fragmentation should be independent by the detailed properties of the string, the conclusion ${ }^{1}$ remains that the rotating string is more likely to fragment into massive pieces than any other change in its motion, including the emission of gravitational radiation. So for both Type I and Type II superstrings, the rotating string will fragment before it can emit an appreciable amount of graviational radiation.

Sivaram's comment that superstrings would not last long enough to form galaxies would apply if superstrings formed galaxies by condensing matter around them as envisaged for galaxy formation by the cosmic strings of Kibble, but is irrelevant to the formation of galaxies by fragmentation of the superstring, where the entire mass of the galaxy comes from the mass of the superstring. As there is no ordinary matter until the hierarchical fragmentation of the superstring is completed, ordinary astrophysical processes, including nucleosynthesis, do not occur until after fragmentation.

Sivaram overestimates the life of a galaxy-forming superstring, as the string has the mass of the galaxy but a much smaller size. A superstring with the mass of a galaxy is about $1 \mathrm{pc}$ in length and so has a lifetime less than 3,000 years.

Superstrings cannot form galaxies by accretion of ordinary matter, but can form galaxies by fragmentation.

\section{J. TASSIE}

Department of Theoretical Physics, Research School of Physical Sciences, The Australian National University, GPO Box 4, Canberra,

\section{Australia}

1. Tassie, L. J. Nature 323, 40-42 (1986).

Witten, B. Phys. Lett. B153, 243-246 (1985).

\section{Run don't walk}

SIR-The conclusion of Ker et al. (Nature $325,147-149 ; 1987)$, that the arch of the human foot stores enough energy to make running more energy efficient, may have been anticipated by Sir Charles Bell. In his book The Hand its Mechanisms and Vital Endowments as Evincing Design, pub-lished in 1833, he wrote that "The spring of the foot and the toe ... gives elasticity and rapidity in running, dancing and leaping". I note that Ker et al. have, as yet, only studied running.

Wessex Neurological Centre,

J.D. Cole

Southampton General Hospital, Shirley, Southampton SO9 $4 X Y$, UK 